Recebido em 10.04.2012. Aprovado em 04.07.2012 Este artigo tem coautoria de membro do Corpo Editorial Científico da RAE foi avaliado em double blind review, com isenção e independência. Editores Científicos: Valter Afonso Vieira, Heitor Takashi Kato, Eliane Pereira Zamith Brito e Lelis Balestrin Espartel

\title{
BRASILEIRO GOSTA DE "MUVUCA"? IMPACTO DA DENSIDADE HUMANA NO COMPORTAMENTO DE COMPRA
}

\author{
DO BRAZILIANS LIKE CROWDING? THE IMPACT OF HUMAN DENSITY ON PURCHASE \\ BEHAVIOR
}

¿AL BRASILEÑO LE GUSTAN LOS AMONTONAMIENTOS? IMPACTO DE LA DENSIDAD HUMANA EN EL COMPORTAMIENTO DEL COMPRADOR

\section{RESUMO}

Este estudo investigou como as variações de densidade humana nas lojas afetam a percepção de crowding e a satisfação dos consumidores. Por meio de dois experimentos, testou-se como a relação entre a densidade e as respostas dos consumidores é moderada pelas variáveis: familiaridade, habilidade de avaliar a estética de uma loja e nível de renda dos consumidores. Os testes revelaram que, enquanto o aumento da familiaridade amortece reações negativas ao crowding, o aumento do nível pers- picácia estética agrava a reação negativa do consumidor. Não houve diferenças significativas nas respostas entre os níveis de baixa e média densidade humana, mas verificou-se aumento das reações negativas na alta densidade. Diferenças nas reações de consumidores de baixa e da alta renda não foram observadas. O trabalho traz sugestões gerenciais e oferece uma contribuição teórica para o melhor entendimento do fenômeno de crowding, e sugere caminhos para a continuidade da sua investigação.

PALAVRAS-ChAVE Densidade humana no varejo, crowding, renda, familiaridade, perspicácia estética.

Marcelo Moll Brandão mollmkt@gmail.com
Professor do Programa de Mestrado e Doutorado, Universidade Nove de Julho - São Paulo - SP, Brasil

Juracy Parente juracy.parente@fgv.br

Professor da Escola de Administração de Empresas de São Paulo, Fundação Getulio Vargas - São Paulo - SP, Brasil

\begin{abstract}
This study investigated how the changes in human density in stores affect the consumers' perception of crowding and satisfaction. Two experiments were conducted to test how shoppers behave in different levels of store crowding, moderated by the variables: familiarity, acumen towards the store aesthetics, and consumers' income. The moderation tests indicated that while higher familiarity reduces the negative responses to crowding, higher levels of aesthetic acumen have an opposite effect. The results indicated non-significant differences in consumers' responses between the low and medium stores human density levels, but showed a substantial increase of negative responses between the medium and high density level. The results indicated non-significant differences between lower and upper income responses. This paper brings not only managerial suggestions, but offers a significant contribution to a better understanding and integration of conceptual frameworks about the crowding phenomenon, and suggests many avenues for further investigation.

keywords Retail human density, crowding, income, familiarity, aesthetic acumen.

Resumen Este estudio investigó cómo la variación de densidad humana en las tiendas afecta la percepción de crowding y la satisfacción de los consumidores. Por medio de dos experimentos, se testeó cómo la relación entre la densidad y las respuestas de los consumidores es moderada por las variables: familiaridad, habilidad de evaluar la estética de una tienda y nivel de renta de los consumidores. Los resultados indicaron diferencias no significativas en las respuestas entre los niveles de baja y media densidad humana, pero revelaron reacciones negativas entre la media y alta densidad. Los tests de moderación de las variables familiaridad y perspicacia de estética indicaron que, mientras el aumento de la familiaridad amortece reacciones negativas al crowding, el aumento del nivel perspicacia estética agrava la reacción negativa del consumidor. El trabajo trae sugerencias gerenciales y ofrece una contribución teórica para el mejor entendimiento del fenómeno de crowding, y sugiere caminos para la continuidad de su investigación.
\end{abstract}

Palabras clave Densidad humana en el comercio minorista, crowding, renta, familiaridad, perspicacia estética. 


\section{INTRODUÇÃO}

Diferentes fatores ou elementos ambientais têm sido investigados como influenciadores do comportamento dos consumidores, tais como espaço físico da loja, forma de apresentação dos produtos, iluminação, música, atmosfera da loja, atratividade física da loja, design (leiaute), fatores sociais, atendimento, entre outros (TURLEY e MILLIMAN, 2000; BAKER e outros, 2002). Um dos vários fatores ambientais que têm sido pesquisados no ambiente varejista é o fenômeno de crowding, por meio de investigações que procuram entender como consumidores reagem em situações de lojas apinhadas de clientes e abarrotadas de produtos (EROGLU, MACHLEIT, BARR, 2005; MACHLEIT, K I ELLARIS, EROGLU, 2000; ROMPAY e outros, 2008).

Crowding, fator relacionado ao ambiente de loja, é reconhecido como um fator ambiental dinâmico, pois o movimento de clientes na loja pode variar ao longo dos vários momentos do dia, diferentemente dos fatores de natureza estática como o espaço físico da loja e equipamentos (BAKER e outros, 2002). A densidade em loja influencia a percepção de todos os outros fatores e pode ser definida com base em duas dimensões observáveis na loja: quantidade de pessoas - densidade humana e produtos e coisas densidade espacial (EROGLU e HARRELL, 1986). Segundo Stokols, Pinter, Schopler (1973) e Averill (1973), a diferença entre os temos densidade e crowding é que o primeiro reflete uma mensuração objetiva do ambiente físico, enquanto o segundo é utilizado para definir a dimensão subjetiva e a consequência emocional da densidade. Devido à dificuldade de encontrar um termo equivalente em português que reflita adequadamente esse segundo construto, ao longo deste trabalho, adotar-se-á o termo densidade como sinônimo de crowding em sua perspectiva objetiva, e percepção de crowding como a resposta emocional do indivíduo à densidade.

Os estudos sobre o fenômeno de crowding têm apresentado resultados divergentes no que tange às respostas dos consumidores. Na sua maioria, os estudos sobre densidade testam as respostas dos consumidores a dois níveis opostos de densidade (alta e baixa) e evidenciam reações negativas, em termos de comportamento de compra e satisfação dos consumidores, em níveis elevados de densidade (EROGLU, MACHLEIT, BARR, 2005; MACHLEIT, KELLARIS, EROGLU, 2000; ROMPAY e outros, 2008). Entretanto, alguns trabalhos recentes, realizados fora dos Estados
Unidos, oferecem uma interpretação oposta em relação ao efeito do crowding, especialmente quando essas investigações foram desenvolvidas em países de diferentes contextos culturais, em que níveis mais altos de densidade humana são mais habituais (PONS e LAROCHE, 2007; CHEBAT e outros, 2008; PAN e SIEMENS, 2011).

A densidade humana na loja é um construto que influencia a percepção de valor e o comportamento dos consumidores, pois fornece uma pista social facilmente acessível para inferir sobre o nível de preço dos produtos da loja (BAKER e outros, 2002; BAKER, GREWAL, PRASURAMAN, 1994). Ambientes de compra vazios podem incorrer em percepção de preços altos e provocar pouca motivação para a compra; por outro lado, lojas cheias podem sugerir preços atrativos, porém podem também indicar uma condição desconfortável, pouco funcional para as compras (PAN e SIEMENS, 2010). Levando em conta essas considerações de vantagens e desvantagens entre os dois níveis opostos de densidade (baixa e alta), pesquisas recentes, buscando melhor entender a relação entre densidade e as respostas dos consumidores, agregaram um nível intermediário, como uma terceira medida de densidade (MICHON e CHEBAT, 2008; PAN e SIEMENS, 2010). Os achados sugerem que os consumidores preferem níveis médios de densidade.

No contexto varejista, pesquisas vêm sendo realizadas para avaliar a influência moderadora no comportamento do consumidor de variáveis demográficas, como o nível de renda dos consumidores (BAKER e outros, 2002; KONUS, VERHOEF, NELSIN, 2008), ou de variáveis situacionais, como o nível de familiaridade do consumidor com o ambiente varejista (HUI e BATESON, 1991; DION, 2004; ROMPAY e outros, 2008), ou de variáveis de personalidade, como a capacidade do consumidor de perceber os detalhes estéticos (perspicácia estética) da loja (FISKE e KINDER, 1980; BEATTIE, 1983; VIEIRA, 2010; HOYER e STOKBURGER-SAUER, 2012). Entretanto, os efeitos dessas variáveis ainda não foram testados como moderadoras da relação entre os diferentes níveis de densidade humana na loja e as respostas dos consumidores.

O presente estudo visa preencher algumas das atuais lacunas no conhecimento sobre o fenômeno de crowding. Os resultados conflitantes dos estudos anteriores, sobre a direção do efeito da densidade nas respostas dos consumidores, serviram de motivação para investigar, no contexto do mercado de um país 
emergente, como o Brasil, as respostas dos consumidores aos diferentes níveis de densidade humana. Buscando ampliar o entendimento sobre esse tema, este estudo irá também explorar, no contexto brasileiro, o efeito moderador das seguintes variáveis, que ainda não foram pesquisadas em estudos anteriores sobre crowding: o nível de renda dos consumidores, a familiaridade com o ambiente varejista e a habilidade do consumidor em avaliar a estética visual de um ambiente de loja (perspicácia estética). Para suportar teoricamente as hipóteses que serão testadas neste estudo, uma breve revisão do conhecimento sobre a influência dessas variáveis no comportamento do consumidor será apresentada na próxima seção.

Este trabalho busca, então, responder às seguintes questões de pesquisa: O que ocorre com a percepção de crowding e satisfação dos consumidores em diferentes condições de densidade humana? Quão diferente é a reação dos consumidores de baixa e alta renda a esses ambientes de compra? E se os consumidores estiverem em diferentes níveis de familiaridade (baixo e alto)? Ou, ainda, qual seria a resposta em relação à percepção de crowding e satisfação de consumidores com baixa e alta habilidade em avaliar a estética visual de um ambiente de loja? Por meio de dois experimentos, este estudo testa as relações entre diferentes níveis de densidade humana e indicadores de comportamento do consumidor, e o efeito de três variáveis moderadoras nessas relações.

\section{ARCABOUÇO TEÓRICO E HIPÓTESES}

Com base no clássico paradigma S-O-R (Estímulo - Or- ganismo - Resposta) de Mehrabian e Russell e apoiado na revisão do conhecimento sobre o fenômeno de crowding, elaborou-se um modelo (Figura 1) que demonstra as relações entre as variáveis envolvidas nas hipóteses desta pesquisa. A variável independente consiste nos níveis de densidade humana encontrados na loja. As variáveis dependentes são as respostas dos consumidores, operacionalizadas por meio das variáveis: percepção de crowding (PC) e satisfação (SAT). O papel de moderação é exercido pelas variáveis: renda (Experimento 1), perspicácia estética (Experimento 1) e familiaridade (Experimento 2).

Entende-se que densidade em loja é um construto que influencia a percepção de valor e comportamento dos consumidores em loja. Essa influência pode ser explicada por meio da teoria de categorização (ALBA e HUTCHINSON, 1987; COHEN e BASU, 1987), a qual propõe que os consumidores utilizam informações avaliáveis para ajudar a inferir sobre informações que não estão acessíveis facilmente, tais como preço e qualidade, com base em pistas acessíveis, comparando com seu conhecimento anterior sobre a situação. As pistas acessíveis no ambiente de loja, segundo Baker (1998) e Baker e outros (2002), são os estímulos que compõem o ambiente de loja.

Donovan e Rossiter (1982) evidenciaram uma relação entre os ambientes de loja, respostas emocionais dos indivíduos e comportamento positivo ou negativo de compras. A estimulação excessiva, que pode ocorrer com alta densidade em um ambiente, poderia levar o consumidor a uma resposta negativa ou, pelo menos, não associada com as suas expectativas (KUMAR, PETERSEN, LEONE, 2010). O aumento da percepção de crowding ocorreria quando o nível de densidade

\section{Figura 1 - Modelo teórico testado}

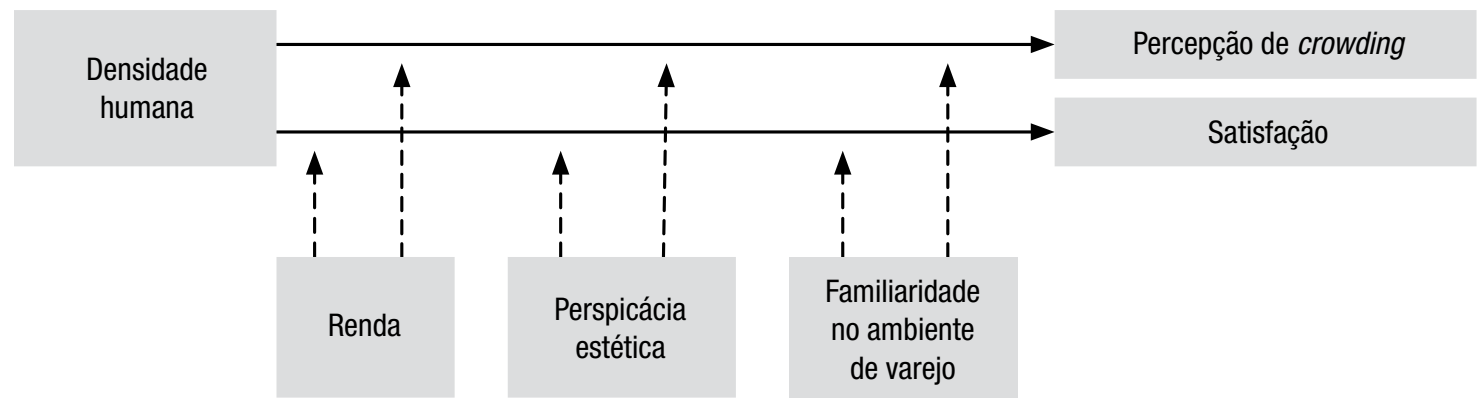


ultrapassasse o nível de tolerância ao crowding do indivíduo ou o impedisse de atingir os seus objetivos. Nesse sentido, o simples incremento da densidade humana em loja, necessariamente, não incrementaria a percepção de crowding.

Várias pesquisas (MACHLEIT, KELLARIS, EROGLU, 1994; EROGLU, MACHLEIT, BARR, 2005; ROMPAY e outros, 2008) indicam que o incremento de densidade humana e espacial aumenta a percepção de crowding e diminui a satisfação dos consumidores. Em todas as pesquisas citadas, a justificativa de uma resposta negativa foi relacionada ao maior nível de stress gerado pelo aumento da densidade humana e espacial. Entretanto, deve-se lembrar que os estudos tradicionais sobre esse fenômeno foram desenvolvidos nos Estados Unidos, em ambientes de varejo com níveis habituais de densidade humana baixa. Pesquisas recentes, realizadas em outros países caracterizados por níveis habituais de densidade humana elevada, indicam reações positivas dos consumidores (PONS e LAROCHE, 2007; PAN e SIEMENS, 2011).

Nos dois grupos de pesquisas, as referências objetivas de densidade foram a quantidade de pessoas em um ambiente varejista, conforme desenvolvido por Machleit, Kellaris, Eroglu (1994). Os resultados conflitantes dos estudos anteriores, sobre a direção do efeito da densidade nas respostas dos consumidores, motivaram a investigação do impacto da densidade humana no contexto do mercado varejista do Brasil. Conforme adotado na maior parte dos estudos anteriores sobre crowding, o Experimento 1 testa o impacto de dois níveis (baixo e alto) de densidades e postula hipóteses alinhadas aos resultados da maioria dos estudos sobre esse tema:

H1: A percepção de crowding (PC) é incrementada com o aumento do nível de densidade humana.

H2: A satisfação (SAT) é incrementada com a diminuição do nível de densidade humana.

É possível que os divergentes resultados sobre o impacto da densidade humana na reação dos consumidores tenham sido ocasionados pelo efeito moderador exercido por diferentes condições ou variáveis ambientais, demográficas, psicográficas ou situacionais. Nesse sentido, espera-se que a inclusão de variáveis moderadoras amplie o entendimento e melhor explique o fenômeno de crowding.

A renda como moderadora da reação dos consumidores já havia sido proposta por artigos ante- riores (BAKER e outros, 2002; KONUS, VERHOEF, NELSIN, 2008), entretanto nenhum dos estudos sobre crowding testou a variável renda como moderadora das respostas dos consumidores aos diferentes níveis de crowding.

Segundo Chauvel e Mattos (2008), a percepção de valor dos consumidores no Brasil é diferenciada entre os segmentos de baixa e alta renda. De fato, os autores do presente artigo, ao observarem ambientes de compra em São Paulo, em diferentes contextos varejistas, verificaram uma grande diferença entre os altos níveis de densidade humana encontrados em polos varejistas de baixa renda (ex: Largo Treze, S. Miguel Paulista), comparados aos menores níveis de densidade humana em polos varejistas voltados para os níveis mais altos de renda, como shopping centers.

O sucesso de polos varejistas congestionados e com altíssima densidade humana, como a famosa Rua 25 de Março, em São Paulo, ou o Beco da Poeira, em Fortaleza, parece sinalizar a aceitação de segmentos da população brasileira para regiões de compra de alta densidade humana (QUEZADO e outros, 2012).

Espera-se que consumidores de baixa renda tenham respostas mais positivas do que consumidores de alta renda à alta densidade humana. Assim, as hipóteses de moderação de renda podem ser enunciadas como:

\footnotetext{
H3: Com o aumento de densidade humana, consumidores com maior renda têm maior percepção de crowding (PC) que consumidores de menor renda. H4: Com o aumento da densidade humana, consumidores com maior renda têm menor satisfação (SAT) do que consumidores com menor renda.
}

O comportamento do consumidor é influenciado pela sua percepção da estética relacionada ao produto ou ao local onde o serviço é oferecido (VIEIRA, 2010; HOYER e STOKBURGER-SAUER, 2012). Reconhece-se que a habilidade de perceber as diferentes dimensões de objetos ou ambientes varia entre diferentes pessoas. Neste trabalho, essa habilidade será denominada "perspicácia da estética". Os indivíduos que têm um maior senso de estética tendem a apresentar maior sensibilidade aos detalhes de certo objeto ou ambiente e a ter maior conhecimento e experiência em relação ao produto ou ao ambiente que está sendo analisado e "experenciado" (BLOCH, 1995; Consumidores com maior conhecimento e experiência apresentam comportamentos diferentes em seus processos decisórios 
de compra quando comparados com os de menor conhecimento. Aqueles com maior expertise processam melhor as informações (BEATTIE, 1983; FISKE e KINDER, 1980), pois conseguem mapear com mais facilidade os estímulos percebidos pela sua mente, desenvolver estruturas cognitivas mais diferenciadas e melhor elaborar as dimensões mais relevantes (ALBA e HUTCHINSON, 1987).

É razoável, portanto, esperar que consumidores com maior nível de "perspicácia da estética" sejam mais sensiveis e consigam melhor discriminar os diferentes níveis de densidade humana na loja. Consequentemente, esses consumidores devem responder mais intensamente aos diferentes níveis de densidade, ou seja, apresentar, em relação aos consumidores com menor "perspicácia da estética", reações mais negativas aos ambientes com níveis mais altos de densidade. Dessa forma, as hipóteses sobre o papel moderador da variável "perspicácia da estética", na relação entre níveis de densidade e respostas dos consumidores, podem ser enunciadas como:

H5: Com aumento da densidade humana, consumidores com maior perspicácia estética (PE) têm maior PC que consumidores com menor perspicácia estética. H6: Com aumento da densidade humana, consumidores com maior PE têm menor satisfação (SAT) que consumidores com menor perspicácia estética.

Em pesquisas anteriores sobre o comportamento do consumidor em loja, traços de personalidade e fatores situacionais surgiram como moderadores da relação entre os diferentes estímulos provocados pelos fatores ambientais de loja e as resposta dos consumidores (HARRELL, HUTT, ANDERSON, 1980; DION, 2004). A familiaridade com o ambiente de loja foi identificada como possível variável moderadora dessa relação, pois a experiência frequente em um ambiente gera maior controle informacional do consumidor sobre as pistas existentes em um ambiente de loja (HUI e BATESON, 1991; DION, 2004; ROMPAY e outros, 2008).

Alguns consumidores apresentam menor controle da situação devido à falta de controle informacional (MACHLEIT, MANTEL, 2001). Ao encontrar-se em uma situação de pouca familiaridade com o ambiente da loja, o consumidor manifestaria um menor controle situacional, maior nível de stress pela falta de controle e um comportamento menos favorável às compras (MACHLEIT, EROGLU, MANTEL, 2000). O sentimento de controle é influenciado pela obstrução ou facilidade de atingir os objetivos. Os estudos de Hui e Bateson (1991) e Rompay e outros (2008) mostraram que menor controle informacional e situacional restringe $\mathrm{O}$ cliente a concretizar seus objetivos de compra. Assim, maior familiaridade em lojas com maior densidade humana faria consumidores apresentarem respostas mais positivas que os com menos familiaridade. Essas considerações permitem a proposição das hipóteses abaixo.

H7: Com aumento da densidade humana em loja, consumidores com menor familiaridade têm maior PC que consumidores com maior familiaridade.

H8: Com aumento da densidade humana em loja, consumidores com menor familiaridade têm menor satisfação (SAT) que consumidores com maior familiaridade.

Pesquisas recentes buscaram um melhor entendimento da relação entre densidade e as respostas dos consumidores, e agregaram um nível intermediário, como uma terceira medida de densidade (MICHON e CHEBAT, 2008; PAN e SIEMENS, 2010). O Experimento 2 irá também testar a reação dos consumidores a três níveis de densidade (baixa, média, e alta).

Os estudos seminais de Stokols, Pinter, Schopler (1973) e Averill (1973) sugerem que o aumento da percepção de crowding ocorreria apenas quando o nível de densidade ultrapassasse o limite de tolerância ao crowding do indivíduo ou impedisse esse indivíduo de atingir seus objetivos. Assim, mesmo que objetivamente o nível de densidade humana fosse incrementado em número suficiente para determinar uma mudança visível de quantidade de consumidores, esse aumento não iria, necessariamente, provocar um aumento da percepção de crowding ou de desconforto e insatisfação do consumidor. Enquanto a estimulação estiver dentro das expectativas dos consumidores, não haveria alteração nas suas respostas emocionais (MASSARA, LIU, MELARA, 2010). Essas considerações sugerem que a relação entre nível de densidade e percepção de crowding seria representada por uma curva em formato de crescimento exponencial. O aumento da percepção de crowding (PC) seria lento entre o nível baixo e nível médio, mas passaria a crescer de maneira acelerada entre o nível médio e alto.

Nas pesquisas realizadas com três níveis de densidade (MICHON e CHEBAT, 2008; PAN e SIEMENS, 2010), a justificativa teórica para resultados mais positivos na média densidade está relacionada à teoria da categorização 
(ALBA e HUTCHINSON, 1987; COHEN e BASU, 1987). Para Loken, Barsalou, Joiner (2008), especificamente nas situações onde o consumidor tem menos informações referentes aos produtos ou não tem incentivo para avaliações mais detalhadas, o ambiente torna-se informação que alimenta os processos heurísticos de decisão do consumidor. Assim, a ausência ou pequeno número de clientes em uma loja poderia sinalizar uma imagem de pouca atratividade. Por outro lado, uma alta densidade humana poderia sugerir experiências de desconforto e stress. Nesse sentido, a relação entre os níveis de densidade e satisfação do consumidor assumiria um formato de U invertido, indicando maiores níveis de satisfação no nível médio de densidade.

Com base nessas considerações, as seguintes hipóteses podem ser enunciadas:

H9: A PC é incrementada somente quando a densidade passa do nível médio para o nível alto.

H10: A SAT é menor nas condições de baixa e alta densidade quando comparada à de média densidade humana.

O Quadro 1 apresenta a operacionalização das variáveis que foram tratadas nos testes desta pesquisa.

\section{EXPERIMENTO 1}

\section{Metodologia do Experimento 1}

Este experimento teve como objetivo testar as Hipóteses $\mathrm{H}_{1}$ a $\mathrm{H}_{6}$ com a manipulação da densidade em dois níveis (baixo e alto). Os testes foram realizados para um experimento com desenho 2 densidade (baixa, alta) x 2 renda (baixa, alta) x 2 perspicácia estética (baixa, alta). Para analisar as diferenças de média entre as condições testadas, foram utilizadas ANOVAS e ANCOVAS. Neste experimento, as análises dos resultados foram realizadas apenas separadamente para as interações de densidade humana e renda, e densidade humana e perspicácia estética conforme prenunciado nas hipóteses desta pesquisa. Neste experimento, as variáveis dependentes foram percepção de crowding e satisfação.

\section{Seleção dos estímulos e coletas de dados}

A manipulação de densidade humana foi realizada com duas fotos de livrarias, sendo uma com duas pessoas no ambiente de loja (baixa densidade humana) e outra como muitas pessoas no ambiente de

\section{Quadro 1 - Variáveis e escalas utilizadas}

\section{Função da \\ variável \\ Variáveis/escalas utilizadas}

Variáveis dependentes

Percepção de crowding: utilizadas as escalas desenvolvidas por Machleit, Kellaris, Eroglu (1994). Neste artigo, foi utilizada escala Likert de sete com quatro questões conforme a escala original.

Satisfação: utilizada a escala Likert de sete pontos, com duas questões conforme a escala original (OLIVER, 1993).

Variável Densidade humana: neste artigo, foi utilizada a escala Likert de sete com duas questões conforme a escala original independente (MACHLEIT, KELLARIS, EROGLU, 1994).

Variável Renda: a variável foi avaliada por meio de faixas de renda com base em estudos anteriores na área de comportamento de moderadora compra e consumo no Brasil (CHAUVEL e MATTOS, 2008).

Variável moderadora

Familiaridade: escala Likert de sete pontos, e operacionalizada com duas questões (DION, 2004).

Variável moderadora
Perspicácia estética: a variável foi avaliada por meio de quatro questões com escalas Likert, desenvolvidas por Vieira (2010). 
loja (alta densidade humana). A escolha por manipular densidade utilizando-se fotos se deu porque essa é a manipulação tradicionalmente utilizada em pesquisa do fenômeno de crowding em ambiente de loja (MACHLEIT, KELLARIS, EROGLU, 1994; PAN e SIEMENS, 2010).

A coleta de dados foi realizada em sala de aula em turmas de marketing de cursos de graduação e pós-graduação em administração de empresas. Os alunos foram apresentados primeiro às questões que mensuravam a sua habilidade ou perspicácia em analisar ambientes em geral. Ao final do experimento, os alunos declararam que não relacionaram as questões de perspicácia estética com a segunda parte do questionário. Após responderem à primeira parte, os alunos foram apresentados às fotos de manipulação de densidade humana em livraria - uma foto de loja com baixa densidade humana apresentada para um grupo, e a foto de loja com alta densidade para o outro grupo. Em seguida, responderam às questões referentes à densidade humana, percepção de crowding, satisfação e sobre uma covariável de avaliação da qualidade dos produtos vendidos na loja. Por fim, eles responderam às questões de renda, sexo, idade.

\section{Método de análise}

As variáveis dependentes foram testadas com base nos seus loadings fatoriais. Os testes foram realizados para as variáveis dependentes PC e SAT. As variáveis independentes, tratadas nos testes como possíveis moderadoras - renda e PE - foram transformadas em variáveis binárias a partir da média dos fatores resumo. Para a variável renda, o nível de rendimento de $\mathrm{R} \$ 2.500,00$ foi adotado para definir baixa renda de alta renda. Esse corte realizado na operacionalização das escalas teve como base o critério de classes de renda da ABEP 2008. Diante da falta de critério único de classificação de classes de renda nos estudos de marketing que consideram renda, realizados no Brasil (BRANDÃO, 2012), o corte com base nos critérios de classe de renda da ABEP 2008 atende às necessidades deste estudo.

\section{Resultados do Experimento 1}

A amostra foi de 70 respondentes, sendo 35\% homens e $65 \%$ mulheres. A idade média foi de 31 anos, sendo a idade mínima 19 e máxima 53 anos, com desvio padrão de 8 anos. Em relação à renda familiar, 40\% são de renda baixa e 60\%, de renda alta. Dos 70 respondentes, $75 \%$ deles são alunos de graduação e $25 \%$, alunos de MBAs de gestão de negócio.

A manipulação de densidade foi aceita $(F=7$, 3 e p-valor $<0,01)$. A covariável foi significante, apresentando p-valor $<0,05$, e assim ela foi mantida nos testes das hipóteses. Dado que os testes do Experimento 1 foram realizados para cada interação separadamente, com desenhos $2 \mathrm{x} 2$ que geraram quatro grupos de análise, a manutenção da covariável também pode ser justificada por meio do teste de máxima covariável. Devido à utilização da covariável, os testes das hipóteses deste experimento foram realizados por meio de modelos de ANCOVA customizados.

Segundo Konus, Verhoef, e Nelsin (2008), as variáveis demográficas idade e sexo influenciam o comportamento do consumidor no varejo. Porém, para os respondentes participantes deste experimento, não existe correlação entre idade e sexo com a percepção de crowding e satisfação. Assim, as variáveis idade e sexo não foram incluídas nos testes deste experimento. Os resultados dos testes da influência do incremento da densidade humana (baixo e alto) na percepção de crowding são maiores na alta densidade do que na baixa densidade $\left(D_{\text {baixa }}=3,10\right.$ vs $D_{\text {alta }}=4,0 \mathrm{com}((1$; 12,11) $\mathrm{F}=10,70 \mathrm{p}$-valor $<0,20)$ ). Entretanto, as diferenças não foram significantes e, assim, a $\mathrm{H}_{1}$ foi rejeitada. Diferentemente, a H2 foi aceita, demonstrando que quando há menor densidade humana os consumidores ficam mais satisfeitos ( $\mathrm{D}_{\text {baixa }}=3,80$ vs $\mathrm{D}_{\text {alta }}=3,13$ $\operatorname{com}((1 ; 8,23) \mathrm{F}=6,34 \mathrm{p}$-valor $<0,01))$.

As hipóteses $\mathrm{H}_{3}$ e $\mathrm{H}_{4}$ não foram aceitas, pois $\mathrm{O}$ teste de efeito interativo da renda versus densidade não foi significante para PC $(2,1,21) \mathrm{F}=1,31 \mathrm{p}$-valor $>0,28)$ nem para SAT. Assim, com base nos resultados desta pesquisa, a inclusão da variável renda não melhorou a explicação do fenômeno de crowding em ambiente de loja. A justificativa pode estar no perfil da amostra composta por alunos de graduação e pós-graduação, que, devido ao seu elevado nível educacional, podem não ser representativos do padrão de respostas da maioria da população de baixa renda. Outro fator que pode ter ocasionado esse resultado pode também estar relacionado com a composição da amostra por faixas de rendimento. A maior parte dos respondentes classificados como baixa renda compunha as faixas de rendimentos próximos ao valor de $\mathrm{R} \$ 2.500,00$, que foi utilizado como divisor entre os dois segmentos de renda. A continuidade da 
investigação do impacto da renda no comportamento do consumidor exigiria novas amostras, com maior cuidado em relação ao balanceamento da quantidade de respondentes de baixa e alta renda nos extremos das faixas de renda.

Os resultados do teste da moderação de perspicácia estética como variável moderadora foram significantes para satisfação $((2,2,08) \mathrm{F}=3,53$ p-valor < 0,05), mas não foram significantes para a percepção de crowding. Na condição de baixa densidade, as médias de satisfação foram $\mathrm{PE}_{\text {baixo }}=3,23$ e $\mathrm{PE}_{\text {alto }}=4,25$ $((1,86,1) \mathrm{F}=2,23 \mathrm{p}$-valor $<0,14)$, e para alta densidade $\mathrm{PE}_{\text {baixo }}=3,24$ e $\mathrm{PE}_{\text {alto }}=3,03,((1,23,1) \mathrm{F}=2,61 \mathrm{p}$-valor $<0,11)$. Ainda para os testes de satisfação como variável dependente, apenas a diferença entre as médias de alta perspicácia estética foram significantes entre as duas condições de densidade humana $\left(\mathrm{DENS}_{\text {baixa }}=\right.$ 4,25 e DENS $_{\text {alta }}=3,03((8,2,1) \mathrm{F}=11,9 \mathrm{p}$-valor $<0,05)$. A variável que capta a habilidade de os indivíduos avaliarem a estética de um ambiente foi evidenciada como mais explicativa da diferença de comportamento dos respondentes que a própria renda, conforme apresentado na Figura 2.

\section{Figura 2 - Gráficos de SAT (condição 1 = baixa densidade e condição 2 = alta densidade) - teste de interação com perspicácia estética (PE)}

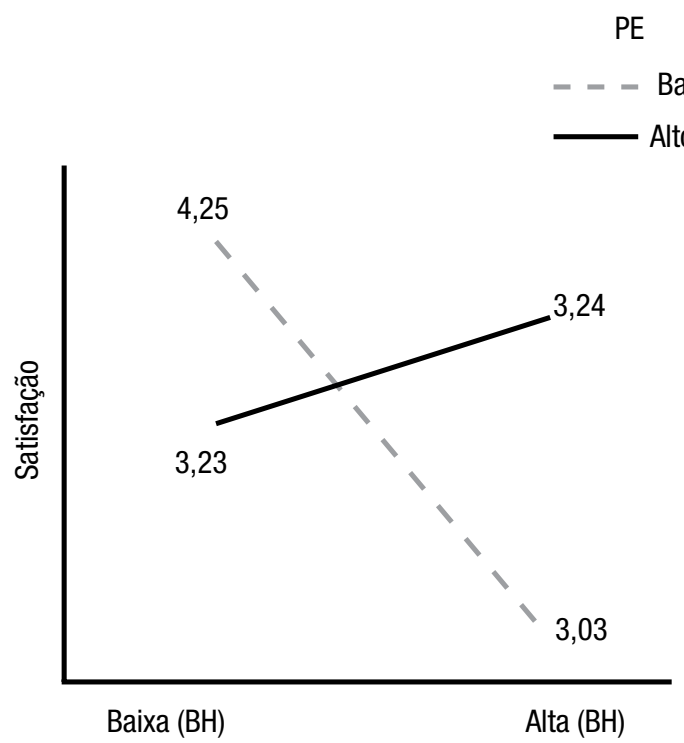

\section{EXPERIMENTO 2}

\section{Metodologia do Experimento 2 e pré-testes}

Este experimento teve como objetivo testar as hipóteses $\mathrm{H}_{7}, \mathrm{H}_{8}, \mathrm{H}_{9}$, e $\mathrm{H}_{10}$. No segundo experimento, os participantes responderam a um questionário on-line. Após avaliarem o estímulo de densidade humana por meio de uma foto do ambiente de loja de uma livraria, cada respondente respondeu às mesmas questões do Experimento 1, mas pela internet, e não em sala de aula, conforme o Experimento 1. Além dos procedimentos de coleta on-line, nesse experimento, em vez de dois níveis de densidade humana, foram tratados três níveis de densidade humana. Foram mantidas as manipulações utilizadas no primeiro experimento, e mais um nível de densidade - média densidade humana. Devido à inclusão de mais um nível de densidade, novos pré-testes foram necessários para verificar se seriam mantidas as fotos do primeiro experimento e qual seria a melhor manipulação para a média densidade humana.

Foram realizados pré-testes com 60 alunos de cursos de administração, com o objetivo de identificar as melhores fotos para manipulação, além de testar o entendimento das escalas que foram traduzidas de pesquisas anteriores sobre o fenômeno de crowding em ambiente de loja. As manipulações foram novamente testadas, porque nesse experimento foi introduzido um nível médio de densidade humana, com mais fotos de livraria. Após o pré-teste, foram mantidas as manipulações de baixa e alta densidade humana, e selecionada uma foto para média densidade.

Para os testes de hipótese das relações diretas e das interações entre as variáveis independentes, utilizaram-se ANOVAs, ANCOVAs e Modelos de Regressão Linear.

\section{Seleção dos estímulos e pré-testes}

As fotos escolhidas no pré-teste foram avaliadas conforme o objetivo das condições de manipulação da pesquisa, ou seja, baixa, média e alta densidade. As manipulações apresentaram diferenças significantes (p-valor < 0,05), e não apresentaram diferenças significantes de percepção de qualidade dos ambientes de loja. Conforme Konus, Verhoef, Nelsin (2008), as respostas dos consumidores à variação do ambiente de loja podem sofrer influência de covariáveis. Para maior controle de variáveis espúrias na realização do experimento, a qualidade percebida foi mensurada e 
tratada como covariável na análise e operacionalizada como "Acho que a loja da foto vende produtos de qualidade". Para testar as igualdades e diferenças de média, foram utilizadas ANOVAs e ANCOVAs.

A manipulação da densidade em loja foi avaliada por meio de ANOVA. Utilizou-se uma escala Likert de 5 pontos para confirmar a manipulação da questão "Tem muita gente na loja da foto" como variável dependente, e como fatores (variáveis independentes) as condições manipuladas de baixa, média e alta densidade. O resultado revelou que existe um efeito principal da diferença de quantidade de pessoas nas lojas, conforme esperado $((\mathrm{F} 2,105)=89,62$ p-valor $<$ $0,01)$. De fato, as condições de baixa, média e alta densidade humana apresentaram respectivamente médias conforme o esperado $\left(D_{\text {baixa }}=1,32\right.$ vs $D_{\text {média }}=2,90$ vs $D_{\text {alta }}=6,23$ com p-valor $\left.<0,01\right)$.

\section{Desenho e método de pesquisa}

O Experimento 2 testou as hipóteses $\mathrm{H}_{9}$ e $\mathrm{H}_{10}$ para as relações diretas entre densidade humana e percepção de crowding e densidade humana e satisfação. Nesse experimento, em vez de dois níveis, serão tratados três níveis de densidade humana. As hipóteses de moderação da renda foram mantidas $\left(\mathrm{H}_{3}, \mathrm{H}_{4}\right)$ conforme $\mathrm{O}$ Experimento 1. As hipóteses $\mathrm{H}_{7}$ e $\mathrm{H}_{8}$ testaram a moderação de familiaridade. O desenho do Experimento 2 pode ser expresso como 3 × 2 × 2 fatorial, onde cada indivíduo foi apresentado a apenas uma condição de densidade. O Experimento 2 foi definido como 3 (baixa/média/alta) densidade humana x 2 (baixa/alta) familiaridade, sendo cada respondente colocado em apenas uma condição de densidade humana e uma única condição de familiaridade. Para análise, foram utilizadas ANOVAs, ANCOVAs e regressão linear para testar as moderações, segundo Baron e Kenny (1986). Neste estudo, as variáveis dependentes utilizadas foram percepção de crowding e satisfação.

\section{Procedimentos e medidas}

Com base em questionários on-line, cada participante respondeu às questões referentes a apenas uma das três condições de densidade. Primeiro, os consumidores foram apresentados a quatro questões que mensuravam a tolerância ao crowding. Apenas uma das fotos foi apresentada a cada um dos três grupos de respondentes. Os respondentes foram recrutados via e-mail, sendo a amostra de conveniência. Os questio- nários foram acessados no site Questionpro por um link colocado nos e-mails de convite enviados aos respondentes.

Após avaliarem as fotos, os respondentes responderam a questões referentes à percepção de crowding, de acordo com os procedimentos adotados por Machleit, Kellaris, Eroglu (1994). A questão "Tem muita gente na loja" serviu como questão de verificação da manipulação de densidade. Na sequência, os participantes responderam a questões de mensuração da satisfação. A seguir, os respondentes responderam a questões de perfil de renda, idade e sexo. Na sequência, os respondentes responderam à mensuração de comportamento do consumidor satisfação e intenção de comprar (probabilidade de comprar na loja). Por fim, foi mensurada a qualidade percebida da loja como covariável conforme o primeiro experimento. Todas as escalas foram discutidas no Quadro 1.

Os testes foram realizados com 105 respondentes. Para validação das medidas dos construtos, foi utilizada uma análise fatorial com base nas respostas obtidas. A validade divergente e convergente das mensurações deu-se por uma análise fatorial exploratória. Os resultados gerais da análise realizada para todas as questões que mensuraram as variáveis dependentes, independentes e moderadoras foram satisfatórios. Foi obtido um teste de KMO significante a 0,01, comunalidades acima de 0,7 para a maioria das questões, e uma variância explicada de 77,4\% para cinco fatores. As questões propostas com base na tradução das questões utilizadas foram corroboradas em função da análise fatorial, mantendo-se as mensurações originais. As variáveis dependentes percepção de crowding e satisfação foram testadas com base em seus loadings fatoriais. A variável familiaridade (FAM), tratada nos testes como possível moderador, foi transformada em variável binária, a partir da média do fator resumo. Da mesma forma que no Experimento 1, as variáveis dependentes foram denominadas PC e SAT.

\section{Resultados do Experimento 2}

A amostra de 105 respondentes do Experimento 2 foi composta de 33\% homens e $67 \%$ mulheres, com idade média de 38 anos e desvio padrão de 14 anos.

As fotos escolhidas no pré-teste foram avaliadas conforme o objetivo das condições de manipulação da pesquisa, ou seja, baixa, média e alta densidade. 
As manipulações apresentaram diferenças significantes ( $p$-valor $<0,05$ ), e não apresentaram diferenças significantes de percepção de qualidade dos ambientes de loja. Conforme Konus, Verhoef, Nelsin (2008), as respostas dos consumidores à variação do ambiente de loja podem sofrer influência de covariáveis. Para maior controle de variáveis espúrias na realização do experimento, a qualidade percebida foi mensurada e tratada como covariável na análise e operacionalizada como "Acho que a loja da foto vende produtos de qualidade". Para testar as igualdades e diferenças de média, foram utilizadas ANOVAs e ANCOVAs.

A manipulação da densidade em loja foi avaliada por meio de ANOVA. Utilizou-se uma escala Likert de 5 pontos para confirmar a manipulação a questão "Tem muita gente na loja da foto" como variável dependente, e como fator (variável independente) as condições manipuladas de baixa, média e alta densidade. O resultado revelou que existe um efeito principal da diferença de quantidade de pessoas nas lojas, conforme esperado $((\mathrm{F} 2,105)=89,62 \mathrm{p}$-valor $<0,01)$. De fato, as condições de baixa, média e alta densidade humana apresentaram respectivamente médias conforme o esperado ( $\mathrm{D}_{\text {baixa }}=1,32$ vs $\mathrm{D}_{\text {média }}=2,90$ vs $\mathrm{D}_{\text {alta }}=6,23 \mathrm{com}$ p-valor $<0,01)$.

Da mesma forma que foram testadas no primeiro experimento, as variáveis sexo e idade não apresentaram correlação significante com a percepção de crowding, mas sim para satisfação. Para nenhum dos resultados houve diferença significante entre os resultados com e sem sexo e idade como covariáveis. Sendo assim, foram adotados os resultados das ANCOVAs apenas com o controle de qualidade percebida da loja para análise dos resultados desse experimento, tanto para percepção de crowding como para satisfação como variáveis dependentes.

No teste de efeitos principais para percepção de crowding nas três condições manipuladas, $\mathrm{a}_{9}$ não foi rejeitada, pois, como proposto na $\mathrm{H}_{9}$, as PC apresentaram mudança significante apenas ao passar de média para a condição de alta densidade humana $((F 2,105)=68,23$ p-valor $<0,01)$. De fato, as médias de PC para baixa e média densidade humana apresentaram diferenças de média não significantes, se considerado o teste de múltiplas comparações de média de Tukey $\left(D_{\text {baixa }}=2,64\right.$ vs $D_{\text {média }}=3,19$, com p-valor $<$ $0,01)$. Apenas a PC para alta densidade foi diferente e significante em relação às outras duas condições $\left(\mathrm{D}_{\text {alta }}\right.$ $=6,0$ p-valor $<0,01)$.

A H10, entretanto, não pode ser confirmada, pois a média densidade não apresentou resultados melhores do que os níveis de baixa e alta densidade. A resposta da variável SAT segue um padrão semelhante de comportamento da PC nas diferentes condições de densidade manipuladas. O resultado de SAT na condição de alta densidade humana $\left(\right.$ Dens $\left._{\text {baixa }}=2,73\right)$ é diferente e significativamente menor em relação às condições de baixa e média densidade $((\mathrm{F} 2,105)=52,70)$. A média de SAT nas condições de baixa e média densidade apresentou diferenças não significantes, se considerado o teste de múltiplas comparações de média de Tukey $\left(D_{\text {baixa }}=4,84\right.$ vs $D_{\text {média }}=4,65$ p-valor $\left.<0,01\right)$. Entretanto, a hipótese propunha que a SAT seria mais alta na média densidade do que na alta. Apenas a condição de alta densidade humana apresentou média diferente e significante $\left(\mathrm{D}_{\text {alta }}=2,73 \mathrm{p}\right.$-valor $\left.<0,01\right)$. $\mathrm{A} \mathrm{H}_{10}$ foi rejeitada, pois não foi possível construir uma curva da relação entre satisfação e densidade humana em forma de U invertido.

As curvas apresentadas na Figura 3 ilustram esses resultados, respectivamente para os testes da $\mathrm{H}_{9}$ e $\mathrm{H}_{10}$. Da direita para a esquerda, são apresentadas, no eixo y, as variáveis dependentes - percepção de crowding e satisfação, e, no eixo x, os três níveis de densidade humana manipulados na pesquisa (baixa, média, e alta).

O teste de interação da variável familiaridade (FAM) com densidade para a PC como variável dependente foi significante $((\mathrm{F} 3,105)=3,53 \mathrm{p}$-valor $<0,05)$, mas com efeito menor que a condição principal densidade $((\mathrm{F} 2,105)=45,39 \mathrm{p}<0,01)$. Essa constatação é reforçada pelos $\mathrm{R} 2$ ajustados dos modelos com e sem a inclusão da moderadora, que respectivamente foram 0,59 e 0,56 . Foi evidenciada, por meio dos parâmetros estimados na ANCOVA, interação com familiaridade apenas na condição de média densidade $\left(\mathrm{FAM}_{\text {baixa }}=\right.$ 4,2 vs $\mathrm{FA}_{\text {Malta }}=2,8$; p-valor $\left.<0,01\right)$. Com base nesse resultado, a hipótese $\mathrm{H}_{7}$ não pode ser rejeitada, pelo menos parcialmente, pois na média densidade humana a diferença entre baixa e alta familiaridade foi significante e houve interação entre as variáveis. O teste de moderação realizado, segundo Baron e Kenny (1986), foi inconsistente, permitindo, assim, apenas a discussão de uma interação entre os níveis de densidade humana e familiaridade.

O teste de interação da variável familiaridade (FAM) com densidade humana para a SAT foi significante $((\mathrm{F} 3,105)=3,93 \mathrm{p}$-valor $<0,10)$, mas com efeito menor que a condição principal, que foram os níveis de densidade $((\mathrm{F} 2,105)=23,38 \mathrm{p}<0,01)$. Os resultados 
indicam, conforme ilustrado na Figura 3, que consumidores com mais familiaridade manifestam menor percepção de crowding e maior satisfação do que os de menor familiaridade. Foi evidenciada, por meio dos parâmetros estimados na ANCOVA, uma interação com familiaridade apenas na condição de média densidade $\left(\mathrm{FAM}_{\text {baixa }}=3,65\right.$ vs $\mathrm{FAM}_{\text {alta }}=5,04$; p-valor < 0,01). Com base nesse resultado, a hipótese $\mathrm{H}_{8}$ não pode ser rejeitada, pois na média densidade humana a diferença entre baixa e alta familiaridade foi significante. O teste de moderação realizado, segundo Baron e Kenny (1986), foi inconsistente, permitindo, assim, apenas a discussão de uma interação entre a densidade e familiaridade. As curvas apresentadas na Figura 4 ilustram esses resultados, respectivamente para os testes da $\mathrm{H}_{7}$ e $\mathrm{H}_{8}$. Da direita para a esquerda, são apresentadas, no eixo y, as variáveis dependentes - percepção de crowding e satisfação, e no eixo x, os três níveis de densidade humana manipulados na pesquisa (baixa, média, e alta), com a influência da moderadora familiaridade.

\section{Teste post-hoc}

Dado que não houve mensuração direta de questões referentes a um maior convívio com situações de maior densidade humana, mas entendendo-se que a familiaridade com o movimento da loja, uma das escalas da variável familiaridade, seria uma proxy para testar de maneira exploratória a relação entre uma interação do nível de renda com o nível de densidade (variáveis independentes) e sua associação com a familiaridade (variável dependente), foi realizado um teste por meio de regressão multivariada. Outra justificativa para um teste exploratório das relações entre essas variáveis seria o fato de elas ainda não terem sido avaliadas em relação ao fenômeno de crowding no contexto de varejo brasileiro. Renda foi uma das variáveis de perfil mensuradas no Experimento 2 e operacionalizada da mesma forma que no Experimento 1.

O resultado do teste por meio de regressão linear confirmou uma interação entre o incremento da densidade humana e renda, e ainda apresentou associação negativa com a familiaridade $((\beta=-0,41, \mathrm{p}$-valor $\leq 0,01)$ $\mathrm{F}=5,8, \mathrm{p}$-valor $\leq 0,01)$. Tais resultados exploratórios permitem uma proposição de maior familiaridade para ambiente de loja com maior densidade humana para consumidores com menor renda.

\section{Conclusões do Experimento 2}

Os resultados relacionados com $\mathrm{H}_{9}$ e $\mathrm{H}_{10}$ indicam que não há alteração da satisfação, ao passar de ambientes de densidade humana baixa para densidade média. Essa situação parece fazer sentido no Brasil, onde consumidores estão mais habituados a conviver com situações de alta densidade humana (QUEZA-

\section{Figura 3 - Gráficos de PC e SAT (condição 1 = baixa densidade; condição 2 = média densidade; e condição 3 = alta densidade)}

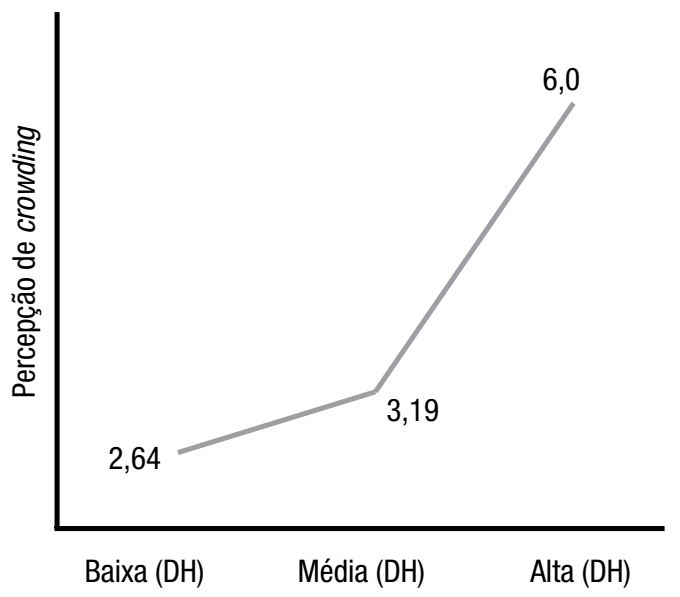


DO, 2012). De uma forma mais abrangente, a maior parte dos consumidores brasileiros, algo em torno de $52 \%$ da população, percebe os serviços públicos de saúde e transportes como ineficientes - infere-se que os consumidores da baixa renda têm como contexto habitual ruas com forte movimento de pedestres, transporte coletivo lotado de passageiros nos horários do rush, trânsito urbano mais congestionado, filas de atendimento mais longas e demoradas em hospitais e postos de saúde (NERI, 2011). Nesse sentido, é razoável que esses consumidores sejam menos sensíveis ao incremento da densidade humana.

Sugere-se, então, que o ambiente habitual com maior densidade humana possa ter influenciado a relação entre densidade humana e satisfação, minimizando a valência negativa da densidade. A variável ambiente habitual, ou mesmo uma operacionalização dessa variável por meio de uma "densidade habitual", não foi medida nem controlada neste experimento, mas poderia justificar a menor influência da densidade humana até o nível de média densidade na satisfação dos respondentes. Uma indicação para essa abordagem foi empiricamente explorada no teste post-hoc, onde foi evidenciada uma interação entre a condição e o nível de renda, que influenciaram a familiaridade com o movimento da loja.

Os resultados indicam que a familiaridade efetivamente exerce uma função moderadora na relação entre o baixo e alto nível de densidade humana e as respostas dos consumidores, pois aqueles com maior nível de familiaridade são mais tolerantes do que os com menor familiaridade, apresentando menor percepção de crowding e maior satisfação.

\section{CONCLUSÕES}

Ao longo deste artigo, conseguimos responder às questões que motivaram essa investigação. O estudo confirmou que variações de densidade humana nas lojas afetam a percepção de crowding e a satisfação dos consumidores. A introdução de três medidas de densidade humana proporcionou um melhor entendimento das respostas dos consumidores aos estímulos ambientais da densidade humana. Os resultados indicaram que os consumidores são pouco sensíveis às variações de densidade humana entre os níveis baixo

\section{Figura 4 - Gráficos de PC e SAT (condição 1 = baixa densidade; condição 2 = média densidade;} e condição 3 = alta densidade) - teste de interação com controle informacional (FAM_D)
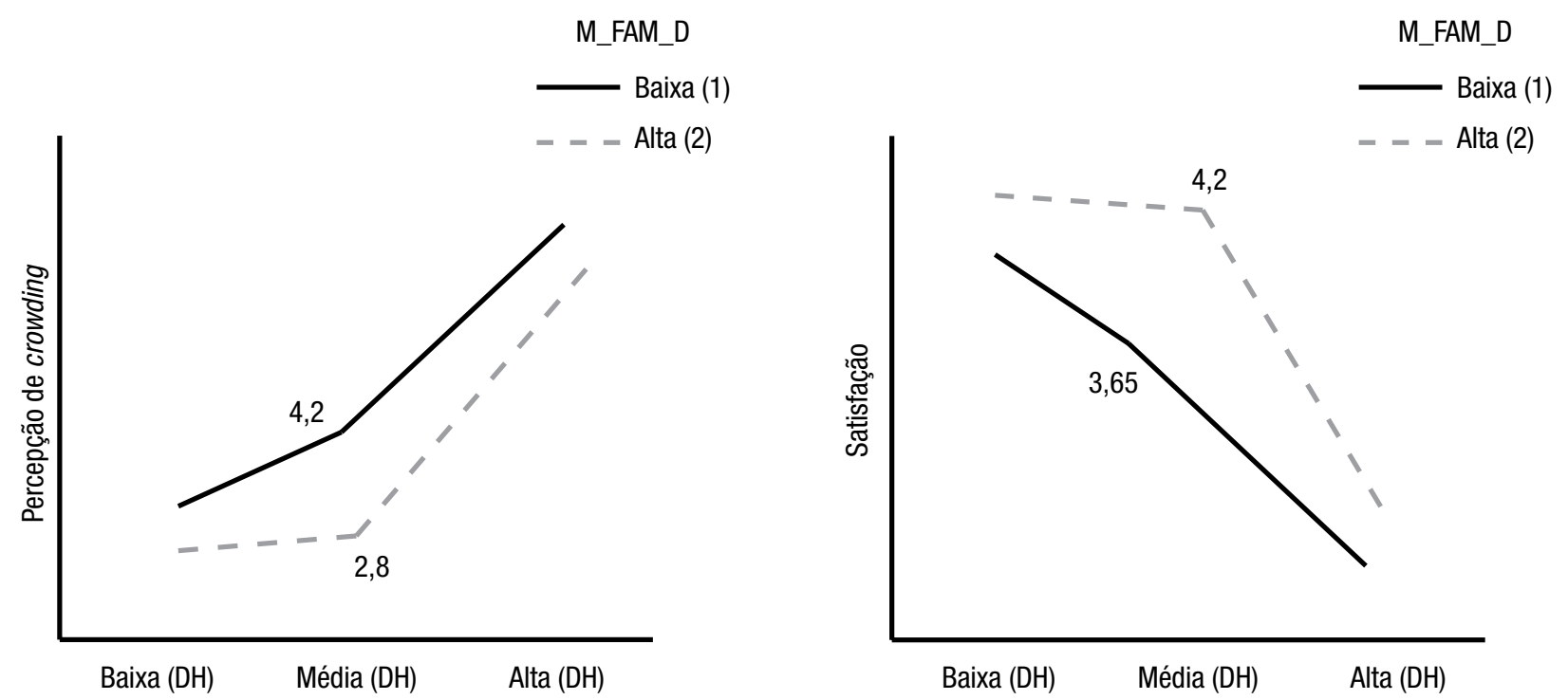

Nota: na legenda de FAM, 1 significa FAMbaixa e 2 FAMalta 
e médio, porém a reação negativa se acentua quando a densidade humana passa do nível médio para o alto.

Os resultados do teste do efeito interativo da renda versus densidade não foi significante nem para percepção de crowding, nem para satisfação. Assim, com base nos resultados desta pesquisa, a inclusão da variável moderadora renda não melhorou a explicação do fenômeno de crowding. A justificativa para esse resultado pode estar no perfil da amostra composta por alunos de graduação e pós-graduação, que, devido ao seu elevado nível educacional, podem não ser representativos do padrão de respostas da maioria da população de baixa renda. Outro fator que pode ter ocasionado esse resultado pode também estar relacionado com a composição da amostra por faixas de rendimento. Nova investigação do impacto da renda no comportamento do consumidor irá exigir um maior cuidado na composição da amostra, para garantir uma maior heterogeneidade de composição entre os grupos de alta e de baixa renda.

Os resultados indicam que a familiaridade efetivamente exerce uma função moderadora na relação entre o baixo e alto nível de densidade humana e as respostas dos consumidores, pois aqueles com maior nível de familiaridade são mais tolerantes do que os com menor familiaridade, apresentando menor percepção de crowding e maior satisfação. Uma análise exploratória evidenciou uma relação negativa entre familiaridade e renda, sugerindo maior familiaridade à alta densidade humana para consumidores da baixa renda. Nos testes de moderação da variável perspicácia de estética, foi possível identificar que elas afetam as respostas dos consumidores. Enquanto o aumento da familiaridade diminui as reações negativas ao crowding, o aumento do nível de perspicácia estética agrava a reação negativa do consumidor.

A evidência da moderação da familiaridade e da perspicácia estética no fenômeno de crowding estimula a continuidade da investigação da moderação de outra dimensão sugerida por Vieira (2010), ou seja, a reação à percepção de valor. A percepção de valor hedônico e utilitário já foi evidenciada como mediadora da relação entre densidade e satisfação (EROGLU, MACHLEIT, BARR, 2005). Um aprofundamento das investigações nesse tema poderia verificar como as percepções de valor diferem entre consumidores de baixa e alta ren$\mathrm{da}$, em diferentes situações de densidade humana em lojas. É possível que consumidores de baixa renda, que recentemente começaram a ter acesso a uma variedade mais ampla em suas cestas de compra, venham a sentir um prazer nessa nova conquista e, portanto, manifestem maior percepção de valor hedônico no processo de compra.

Os resultados deste trabalho não podem ser generalizados devido às amostras terem sido pequenas e, ainda, por elas serem compostas apenas por estudantes de graduação e MBAs. Os testes da moderação da renda podem ter sido prejudicados devido à amostra não ter apresentado uma composição com maior diferenciação de renda entre os dois segmentos (alto e baixo).

Como implicações gerenciais, o estudo ressalta a necessidade de um melhor gerenciamento para manter níveis toleráveis de densidade humana no ambiente de loja, por meio do planejamento mais efetivo para agilizar o atendimento na loja e, assim, atenuar o sentimento de desconforto.. Desenvolver uma comunicação interna e um visual merchandising mais adequado permitirá também um maior controle informacional, o que deverá ampliar o nível de tolerância do consumidor a situações de alta densidade humana.

Próximas pesquisas poderiam, além de ampliar o entendimento sobre o efeito de variáveis moderadoras, incorporar investigações sobre o impacto da interação entre densidade humana e espacial nas respostas dos consumidores e como essas reações diferem entre os diversos segmentos econômicos. A realização de estudos com outros setores varejistas poderá identificar novos padrões de comportamento e reações às situações de crowding.

\section{REFERÊNCIAS}

ALBA, J. W; HUTCHINSON, W. J. Dimensions of consumer expertise. Journal of Consumer Research, v. 13, n. 4, p. 411-454, 1987.

AVERILL, J. R. Personal control over aversive stimuli and its relationship to stress. Psychological Bulletin, v. 80, n. 4, p. 286-303, 1973.

BAKER, J. Examining the informational value of store environments. In: Sherry, J. F. Service scapes. Chicago: NTC/ Contemporary Publishing, 1998. p. 55-79.

BAKER, J. e outros. The influence of multiple store environments use on perceived merchandise value and patronage intentions, Journal of Marketing, v. 66, n. 2, p. 120-141, 2002. 
BAKER, J; GREWAL, D; PARASURAMAN, A. The influence of store environment on quarterly inferences and store image. Journal of the Academy of Marketing Science, v. 22, n. 4, p. 328-339, 1994.

BARON, R.M; KENNY, D.A. The moderator-mediator variable distinction in social psychological research: conceptual, strategic, and statistical considerations. Journal of Personality and Social Psychology, v.51, n.6, p.11731182, 1986.

BEATTIE, A. E. Product expertise and advertising persuasiveness. Advances in Consumer Research, v. 10, p. 581-584, 1983.

BLOCH, P. H. Seeking the ideal form: product design and consumer response. Journal of Marketing, v. 59, n. 3, p. 16-29, 1995.

BRANDÃO, M. M. Crowding no varejo: diferenças na satisfação e percepção de valor hedônico de consumidores de alta e baixa renda no Brasil. 2012. Tese de Doutorado em Administração de Empresas, Escola de Administração de Empresas da Fundação Getulio Vargas, São Paulo, 2012.

CHAuvel, M. A; MATTOS, M. P. A. Z. Consumidores de baixa renda: uma revisão dos achados de estudos feitos no Brasil. Cadernos EBAPE, v. 6, n. 2, p. 17, 2008.

CHEBAT, J. e outros. The influence of mall environment on female fashion shoppers' value and behavior. Journal of Fashion Marketing and Management, v. 12, n. 4, p. 456-468, 2008.

COHEN, J. B; BASU, K. Alternative models of categorization: toward a contingent processing framework. Journal of Consumer Research, v. 13, n. 4, p. 455-473, 1987.

DION, D. Personal control and coping with retail crowding. International Journal of Service Industry Management, $\mathrm{v}$. 15, n. 3, p. 250-263, 2004.

DONAVAN, R. J; ROSSITER, J. R. Store atmosphere: an environmental psychology approach, Journal of Retailing, v. 58, n. 1, p. 34-57, 1982.

EROGLU, S. A; HARRELL, G. D. Retail crowding: theoretical and strategic implications. Journal of Retailing, v. 62, n. 4, p. 349-362, 1986.
EROGlu, S. A; MACHLEIT, K; G. D; BARR, T. F. Perceived retail crowding and shopping satisfaction: the role of shopping values. Journal of Business Research, v. 58, n. 8, p. 1146-1153, 2005.

FISKE, S. T; KINDER, D. R. Involvement, expertise, and schema use: evidence from political cognition. In: Canter, $\mathrm{N}$; Kihstrom, J. (Eds). Personality, cognition, and social interaction. Hillsdale: Lawrence Erlbaum, 1980. p. 171-190.

HARRELl, G. D; HUTT, M. D; ANDERSON, J. C. Path analysis of buyer behavior under conditions of crowding. Journal of Marketing Research, v. 17, n. 1, p. 45-51, 1980

HOYER, W. D; Stokburger-Sauer, N. E. The role of aesthetic taste in consumer behavior. Journal of the Academy of Marketing Science, v. 40, n.1, p. 167-180, 2012.

HUI, M. K; BATESON, J. E. G. Perceived control and the effects of crowding and consumer choice on the service experience. Journal of Consumer Research, v. 1 1, n. 2, p. 174-184, 1991.

KONUS, U; VERHOEF, P; NESLIN, S. Multichannel shopper segments and their covariates. Journal of Retailing, v. 84, n. 4, p. 398-413, 2008.

KUMAR, V; PETERSON, J. A; LEONE, R. P. Driving profitability by encouraging customer referrals: who, when, and how. Journal of Marketing, v. 74, n. 1 p. 1-17, 2010.

LOKEN, B; BARSALOU, L. W; JOINER, C. Categorization theory and research in consumer psychology. In: HAUGTVEDT, C. P; HERR, P. M; KARDES, F. R. Handbook of consumer psychology. Philadelphia: Psychology Press, 2008. p. 133-163.

MACHLEIT, K; KELLARIS, J. J; EROGLU, S. A. Human versus spatial dimensions of crowding perceptions of crowding perceptions in retail environments: a note measurement and effect on shipper satisfaction. Marketing Letters, v. 5, n. 2, p. 183-194, 1994.

MACHLEIT, K; KELlARIS, J. J; EROGLU, S. A. Perceived retail crowding and shopping satisfaction: what modifies this relationship? Journal of Consumer Psychology, v. 9, n. 1, p. 29-42, 2000.

MACHLEIT, K, MANTEL, S. P. Emotional response and shopping satisfaction Moderating effects of shopper 
attributions. Journal of Business Research, v. 54, n. 2, p. 97-106, 2001.

MASSARA, F; LIU, S. S; MELARA, R. D. Adapting to a retail environment: modeling consumer-environment interactions. Journal of Business Research, v. 63, n. 7, p. 673-681, 2010.

MICHON, R; CHEBAT, J. C. Breaking open consumer behavior black box: SEM and retail atmospheric manipulations. Journal of Marketing Theory and Practice, v. 16, n.4, p. 299-307, 2008.

NERI, M. A nova classe média: o lado brilhante da base da pirâmide. São Paulo: Saraiva, 2011.

OLIVER, R. L. Cognitive, affective, and attribute bases of the satisfaction response. Journal of Consumer Research, v. 20, n. 3, p. 418-430, 1993.

PAN, Y; SIEMENS, J. C. The differential effects of retail density: an investigation of goods versus service settings. Journal of Business Research, n. 64, n. 2, p. 105-112, 2011.

PONS, F; LAROCHE, M. Cross-cultural differences in crowd assessment. Journal of Business Research, v. 60, n. 3, p. 269-276, 2007.

QUEZADO, I. e outros. Percepção de crowding e sua relação com valor e local de compra: um estudo em dois centros de consumo de baixa renda. In: Encontro de Marketing da Associação nacional de pós-graduação e pesquisa em administração, 5, Curitiba, 2012. Anais. ANPAD, Curitiba, 2012.

ROMPAY, T. J. L. e outros. Human and spatial dimensions of retail density: revisiting the role of perceived control. Psychology E Marketing, v. 25, n. 4, p. 319-335, 2008.

STOKOLS, D; PINTER, R. M; SCHOPLER, J. Physical, social, and personal determinants of the perception of crowding. Environment and Behavior, v. 5, n. 1, p. 87-115, 1973.

TURLEY, L. W; MILLIMAN, R. E. Atmospheric effects on shopping behavior: a review of the experimental evidence. Journal of Business Research, v. 49, n. 2, p. 193-211, 2000.

VIEIRA, V. A. Visual aesthetics in store environment and its moderating role on consumer intention. Journal of Consumer Behaviour. v. 9, n.5, p. 364-380, 2010. 